Y. Sano

Nagoya Math. J.

Vol. 166 (2002), 183-207

\title{
ON PURELY PERIODIC BETA-EXPANSIONS OF PISOT NUMBERS
}

\author{
YUKI SANO
}

\begin{abstract}
We characterize numbers having purely periodic $\beta$-expansions where $\beta$ is a Pisot number satisfying a certain irreducible polynomial. The main tool of the proof is to construct a natural extension on a $d$-dimensional domain with a fractal boundary.
\end{abstract}

\section{$\S 1$. Introduction}

Let $\beta>1$ be a real number and let $T_{\beta}$ be the $\beta$-transformation on the unit interval $[0,1)$ given by

$$
T_{\beta} x=\beta x-[\beta x],
$$

where $[x]$ denotes the integer part of $x$. Then every $x \in[0,1)$ can be written as

$$
x=\sum_{k=1}^{\infty} b_{k} \beta^{-k}, \quad b_{k}=\left[\beta T_{\beta}^{k-1} x\right] .
$$

We call this representation in base $\beta$ the $\beta$-expansion, which was introduced by Rényi [16]. It is denoted by

$$
x=. b_{1} b_{2} \ldots .
$$

A real number $x \in[0,1)$ is said to have an eventually periodic $\beta$ expansion with period $p$ if there exist integers $m \geq 0$ and $p \geq 1$ such that

$$
x=. b_{1} b_{2} \ldots b_{m}\left(b_{m+1} b_{m+2} \ldots b_{m+p}\right)^{\infty},
$$

where $w^{\infty}$ will denote the sequence $w w w \ldots$. In particular, if we can choose $m=0$, we say that $x$ has a purely periodic $\beta$-expansion with period $p$, that is,

$$
x=.\left(b_{1} b_{2} \ldots b_{p}\right)^{\infty} .
$$

Received February 1, 2000.

1991 Mathematics Subject Classification: 34C35, 58F22. 
We know that $x$ has a purely periodic $\beta$-expansion with period $p$ if and only if $T_{\beta}^{p} x=x$.

For $x=1$, we can define the $\beta$-expansion of 1 in the same way:

$$
d(1, \beta)=. t_{1} t_{2} \ldots, \quad t_{k}=\left[\beta T_{\beta}^{k-1} 1\right] .
$$

Let $D_{\beta}$ be the set of $\beta$-expansions of numbers in $[0,1)$. Parry characterized the set $D_{\beta}$ in [13]. By $<_{\text {lex }}$ will be denoted the lexicographical order, that is, $\left(v_{i}\right)_{i=1}^{\infty}<_{l e x}\left(w_{i}\right)_{i=1}^{\infty}$ means that there exists $k \geq 1$ such that $v_{j}=w_{j}$ for any $1 \leq j<k$ and $v_{k} \lesseqgtr w_{k}$. The (one-sided) shift $\sigma_{s}$ maps a point $\left(v_{i}\right)_{i=1}^{\infty}$ to the point $\left(v_{i}^{\prime}\right)_{i=1}^{\infty}=\sigma_{s}\left(\left(v_{i}\right)_{i=1}^{\infty}\right)$ whose $i$ th coordinate is given by $v_{i}^{\prime}=v_{i+1}$.

TheOREM (PARRY). Let $\beta>1$ be a real number, and let $d(1, \beta)=$ $t_{1} t_{2} \ldots$. Let $w$ be an infinite sequence of positive integers.

(1) If $d(1, \beta)$ is infinite,

$$
w \in D_{\beta} \Longleftrightarrow \forall u \geq 0, \sigma_{s}^{u}(w)<_{\text {lex }} d(1, \beta) .
$$

(2) If $d(1, \beta)$ is finite, $d(1, \beta)=. t_{1} \ldots t_{n-1} t_{n}$, say, then

$$
w \in D_{\beta} \Longleftrightarrow \forall u \geq 0, \sigma_{s}^{u}(w)<_{l e x} d^{*}(1, \beta)=\left(t_{1} \ldots t_{n-1}\left(t_{n}-1\right)\right)^{\infty} .
$$

Bertrand [3] and K. Schmidt [18] investigated eventually periodic $\beta$ expansions. A Pisot number is an algebraic integer $(>1)$ whose conjugates other than itself have modulus less than one. Let $\mathbb{Q}(\beta)$ be the smallest extension field of rational numbers $\mathbb{Q}$ containing $\beta$.

Theorem (Bertrand, K. Schmidt). Let $\beta$ be a Pisot number and let $x$ be a real number in $[0,1)$. Then $x$ has an eventually periodic $\beta$ expansion if and only if $x \in \mathbb{Q}(\beta)$.

In [1], Akiyama gives a sufficient condition for pure periodicity where $\beta$ belongs to a certain class of Pisot numbers. Hara and Ito characterized purely periodic modified $\beta$-expansions for a quadratic irrational number $\beta$ in [8]. The present author studied necessary and sufficient condition for pure periodicity in [11] where $\beta$ is a cubic Pisot number whose minimal polynomial is given by

$$
\operatorname{Irr}(\beta)=x^{3}-k_{1} x^{2}-k_{2} x-1, \quad k_{1}(\neq 0), k_{2} \in \mathbb{N} \cup\{0\}, \text { and } k_{1} \geq k_{2} .
$$




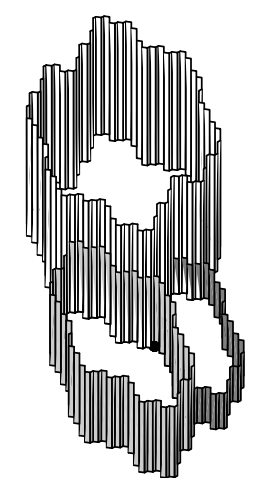

Figure 1: Figure of $\widehat{Y}$ in case $d=3$.

In this paper, we will generalize the results of [11]. Hereafter, $\beta$ is a positive root of the polynomial:

$$
\begin{aligned}
\operatorname{Irr}(\beta)=x^{d}-k_{1} x^{d-1}-k_{2} x^{d-2}-\cdots-k_{d-1} x-1 & \\
k_{i} & \in \mathbb{Z}, \text { and } k_{1} \geq k_{2} \geq \cdots \geq k_{d-1} \geq 1 .
\end{aligned}
$$

Then $\beta$ is a Pisot number. We have the following result:

Main Theorem. Let $x$ be a real number in $\mathbb{Q}(\beta) \cap[0,1)$. Then $x$ has a purely periodic $\beta$-expansion if and only if $x$ is reduced.

We define reduced numbers in Section 5. For our purpose, we introduce a $d$-dimensional domain $\widehat{Y}$ with a fractal boundary (see Figure 1 and the definition in Section 4) and a natural extension of $T_{\beta}$ on $\widehat{Y}$, which were originally discussed in [14] and [19]. In [8] and [9], you can find the basic idea of the proof.

I would like to thank Professor S. Akiyama and Professor Sh. Ito for many helpful suggestions and encouragement. I am grateful to N. Tangiku for a life filled with love and support. Finally, I am greatly grateful to the referee for careful reading the manuscript and giving helpful comments.

\section{$\S 2$. Admissible sequences of $\beta$-expansions}

Recall that $\beta$ is a positive root of the irreducible polynomial

$$
\begin{aligned}
\operatorname{Irr}(\beta)=x^{d}-k_{1} x^{d-1}-k_{2} x^{d-2}-\cdots-k_{d-1} x-1 \\
k_{i} \in \mathbb{Z}, \text { and } k_{1} \geq k_{2} \geq \cdots \geq k_{d-1} \geq 1 .
\end{aligned}
$$


From [4], we know that $\beta$ is a Pisot number. From Theorem (Parry) it follows that

$$
d(1, \beta)=. k_{1} k_{2} \ldots k_{d-1} 1
$$

Let

$$
\beta=\beta^{(1)}, \beta^{(2)}, \ldots, \beta^{\left(r_{1}\right)}
$$

be the real Galois conjugates and

$$
\beta^{\left(r_{1}+1\right)}, \overline{\beta^{\left(r_{1}+1\right)}}, \beta^{\left(r_{1}+2\right)}, \overline{\beta^{\left(r_{1}+2\right)}}, \ldots, \beta^{\left(r_{1}+r_{2}\right)}, \overline{\beta^{\left(r_{1}+r_{2}\right)}}
$$

be the complex Galois conjugates of $\beta$, where $r_{1}+2 r_{2}=d$ and $\bar{v}$ is the complex conjugate of a complex number $v$. The corresponding conjugates of $x \in \mathbb{Q}(\beta)$ are also denoted by

$$
x=x^{(1)}, \ldots, x^{\left(r_{1}\right)}, x^{\left(r_{1}+1\right)}, \overline{x^{\left(r_{1}+1\right)}}, \ldots, x^{\left(r_{1}+r_{2}\right)}, \overline{x^{\left(r_{1}+r_{2}\right)}} .
$$

Let $M$ be the companion matrix of the polynomial (2.1), that is,

$$
M=\left[\begin{array}{ccccc}
k_{1} & k_{2} & \ldots & k_{d-1} & 1 \\
1 & 0 & \ldots & 0 & 0 \\
0 & 1 & \ldots & 0 & 0 \\
\vdots & \vdots & \ddots & \vdots & \vdots \\
0 & 0 & \ldots & 1 & 0
\end{array}\right]
$$

We know that $M$ is a $d \times d$ integer matrix with determinant $(-1)^{d-1}$. It is easily checked that the matrix $M$ is irreducible. Here a nonnegative matrix $A$ is irreducible if for each ordered pair of indices $I, J$, there exists some $n \geq 0$ such that $A_{I J}^{n}>0$, where $A_{I J}$ means the $(I, J)$-element of the matrix $A$. An eigenvector $\boldsymbol{\alpha}$ corresponding to the eigenvalue $\beta$ of $M$ and an eigenvector $\gamma$ corresponding to $\beta$ of the transpose of $M$ are vectors $\boldsymbol{\alpha}={ }^{t}\left[\alpha_{1}, \alpha_{2}, \ldots, \alpha_{d}\right]$ and $\gamma={ }^{t}\left[\gamma_{1}, \gamma_{2}, \ldots, \gamma_{d}\right]$, satisfying

$$
M \boldsymbol{\alpha}=\beta \boldsymbol{\alpha} \text { and }{ }^{t} M \boldsymbol{\gamma}=\beta \gamma, \quad \text { respectively, }
$$

where $t$ indicates the transpose. From the Perron-Frobenius theory, irreducibility implies both eigenvectors are positive. We normalize $\boldsymbol{\alpha}$ and $\boldsymbol{\gamma}$ by putting $\gamma_{1}=1$ and choosing $\alpha_{i}(1 \leq i \leq d)$ to satisfy $\langle\boldsymbol{\alpha}, \gamma\rangle=1$, where $\langle$,$\rangle denotes the standard inner product. By using (2.2), we can see that$ $\alpha_{i}$ and $\gamma_{i}(1 \leq i \leq d)$ are given by

$$
\alpha_{i}=\beta^{1-i} / \sum_{n=0}^{d-1} \beta^{-n} T_{\beta}^{n} 1,
$$




$$
\begin{aligned}
\gamma_{1} & =1=. k_{1} k_{2} \ldots k_{d-1} 1 \\
\gamma_{2} & =T_{\beta} 1=. k_{2} \ldots k_{d-1} 1, \\
& \vdots \\
\gamma_{d-1} & =T_{\beta}^{d-2} 1=. k_{d-1} 1 \\
\gamma_{d} & =T_{\beta}^{d-1} 1=.1=\frac{1}{\beta} .
\end{aligned}
$$

By $\mathbb{Z}[\beta]$ will be denoted the set of polynomials in $\beta$ with integral coefficients. Then both $\left\{\alpha_{1}, \ldots, \alpha_{d}\right\}$ and $\left\{\gamma_{1}, \ldots, \gamma_{d}\right\}$ generate $\mathbb{Z}[\beta]$ and both are bases of $\mathbb{Q}(\beta)$.

It follows from either (2.4) or Theorem (Parry) in Section 1 that a sequence $\left(b_{i}\right)_{i=1}^{\infty} \in D_{\beta}$ if and only if for all $i$

$$
0 \leq b_{i} \leq k_{1}
$$

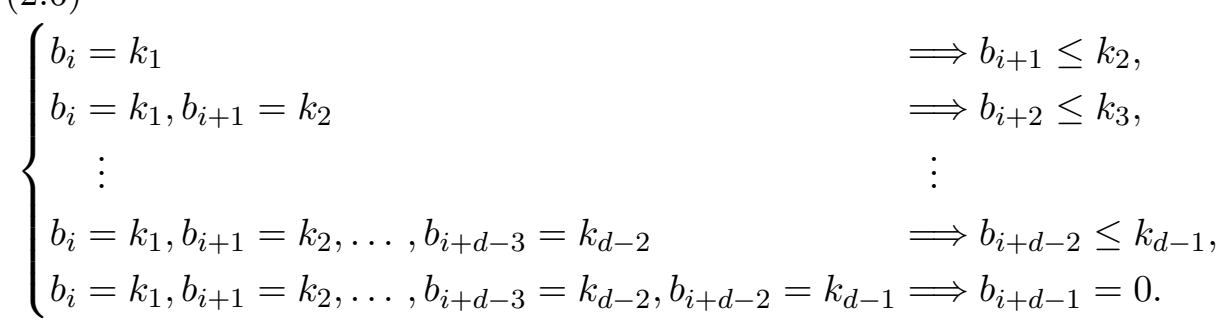

Thus $D_{\beta}$ is represented by the labeled graph $\mathcal{G}$ in Figure 2. In other words, the admissible sequence $\left(b_{i}\right)_{i=1}^{\infty}$ of $\beta$-expansions is an infinite label of the walk in the sofic shift $X_{\mathcal{G}}$. See [12] concerning a labeled graph and a sofic shift.

\section{$\S 3$. Substitutions}

Let $\sigma$ be the substitution of the alphabet $\mathcal{A}=\{1,2, \ldots, d\}$ given by:

$$
\begin{aligned}
\sigma: 1 & \longrightarrow \underbrace{1 \ldots 1}_{k_{1}} 2 \\
2 & \longrightarrow \underbrace{1 \ldots 1}_{k_{2}} 3 \\
d-1 & \longrightarrow \underbrace{1 \ldots 1}_{k_{d-1}} d
\end{aligned}
$$




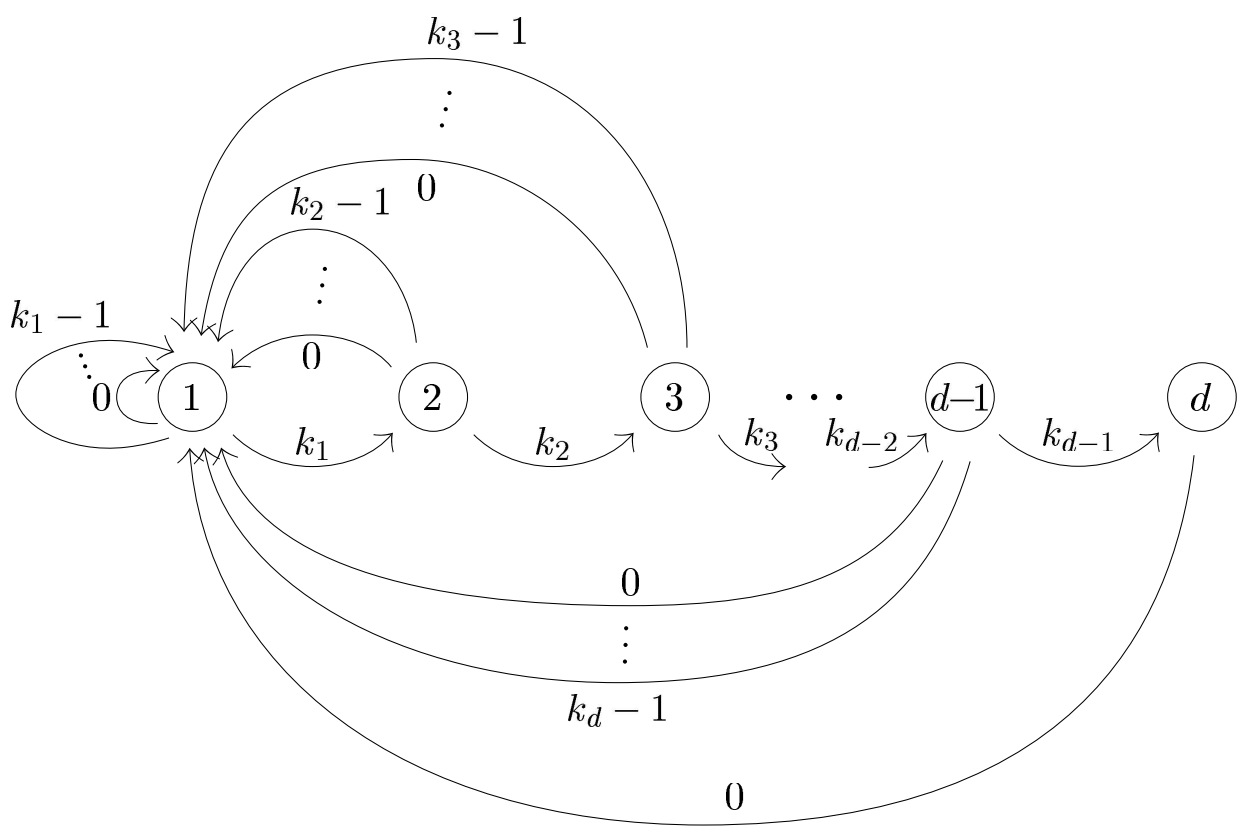

Figure 2: Labeled graph $\mathcal{G}$.

$$
d \longrightarrow 1
$$

The free monoid on $\mathcal{A}$, that is to say, the set of finite words on $\mathcal{A}$, is denoted by $\mathcal{A}^{*}=\bigcup_{n=0}^{\infty} \mathcal{A}^{n}$.

There is a natural homomorphism (abelianization) $f: \mathcal{A}^{*} \rightarrow \mathbb{Z}^{d}$ given by $f(i)=\mathbf{e}_{i}$ for any $i \in \mathcal{A}$ where $\left\{\mathbf{e}_{1}, \ldots, \mathbf{e}_{d}\right\}$ is the canonical basis of $\mathbb{R}^{d}$. Then there exists a unique linear transformation ${ }^{0} \sigma$ satisfying the following commutative diagram:

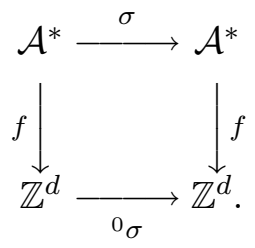

We know that ${ }^{0} \sigma$ is given by the matrix $M$ in Section 2 in our case.

Let $\mathcal{P}$ be the contractive invariant plane of $M$, that is,

$$
\mathcal{P}=\left\{\mathbf{x} \in \mathbb{R}^{d} \mid\langle\mathbf{x}, \gamma\rangle=0\right\}
$$




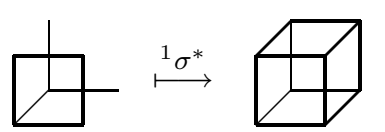

$\left(\mathbf{0}, 1^{*}\right)$

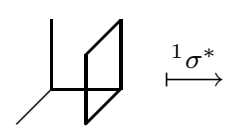

$\left(\mathbf{0}, 2^{*}\right)$
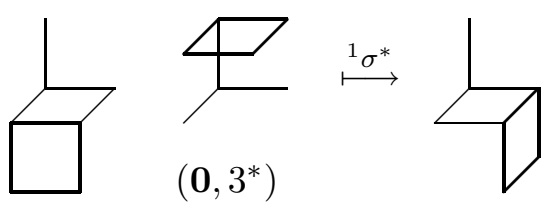

Figure 3: The figure for ${ }^{1} \sigma^{*}$ for the Rauzy fractal $\left(k_{1}=k_{2}=1\right)$.

Let $\pi: \mathbb{R}^{d} \rightarrow \mathcal{P}$ be the projection along the eigenvector $\boldsymbol{\alpha}$. In [15], Rauzy constructed a curious compact domain with a fractal boundary, called the Rauzy fractal, by using the Pisot number $\beta$ for which $\operatorname{Irr}(\beta)=x^{3}-x^{2}-$ $x-1\left(k_{1}=k_{2}=1\right)$. Arnoux and Ito in [2] showed that for any Pisot substitution $\sigma$ a compact domain $X$ with a fractal boundary can be similarly constructed, using the following mapping ${ }^{1} \sigma^{*}$ :

$$
{ }^{1} \sigma^{*}\left(\mathbf{x}, i^{*}\right)=\sum_{j=1}^{d} \sum_{W_{n}^{(j)}=i}\left(M^{-1}\left(\mathbf{x}-f\left(P_{n}^{(j)}\right)\right), j^{*}\right),
$$

where $\sigma(j)=W_{1}^{(j)} \cdots W_{l_{j}}^{(j)}, W_{n}^{(j)} \in\{1, \ldots, d\}, P_{n}^{(j)}$ is the prefix of the letter $W_{n}^{(j)}$, and $\left(\mathbf{x}, i^{*}\right)$ is the set $\left\{\mathbf{x}+\mathbf{e}_{i}+\sum_{j \neq i} \lambda_{j} \mathbf{e}_{j} \mid \lambda_{j} \in[0,1]\right\}$. (See Figure 3.) We remark that we use the notation ${ }^{1} \sigma^{*}$ in stead of $E_{1}^{*}(\sigma)$ which was used in [2].

In [17], the authors define higher dimensional extensions ${ }^{k} \sigma(1 \leq k \leq d)$ of $\sigma$, acting on formal sums of weighted $k$-dimensional faces of unit cubes with vertices in $\mathbb{Z}^{d}$, and their dual maps ${ }^{k} \sigma^{*}$. Moreover, they proved that these maps commute with the natural boundary morphisms and establish some basic properties.

TheOREM. The following limit sets exist in the sense of Hausdorff metric:

$$
\begin{aligned}
X_{i} & :=\lim _{n \rightarrow \infty} M^{n}\left(\pi\left({ }^{1} \sigma^{* n}\left(\mathbf{0}, i^{*}\right)\right)\right) \\
& =\lim _{n \rightarrow \infty} M^{n}\left(\pi\left({ }^{1} \sigma^{* n}\left(-\mathbf{e}_{i}, i^{*}\right)\right)\right), \quad(1 \leq i \leq d) \\
X & =\bigcup_{i=1}^{d} X_{i} .
\end{aligned}
$$

$X_{i}$ are bounded, closed, and disjoint, up to a set of measure 0 . 


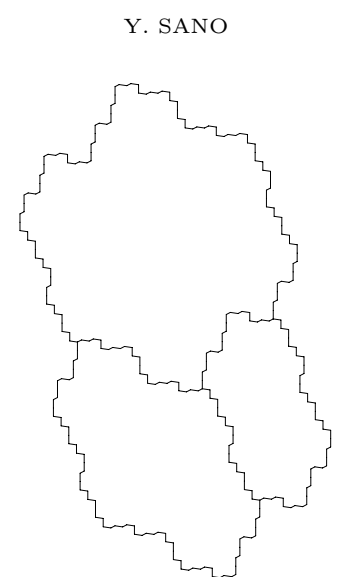

Figure 4: The Rauzy fractal $\left(k_{1}=k_{2}=1\right)$.

Note that the origin of $\mathbb{R}^{d-1}$ belongs to $X$. (See the details in [2].) See Figure 4 in case $k_{1}=k_{2}=1$.

From the equation (3.1) and $M^{-1} \mathbf{e}_{1}=\mathbf{e}_{d}$, we see that the mapping ${ }^{1} \sigma^{*}\left(\mathbf{0}, i^{*}\right)(1 \leq i \leq d)$ in our case are given by

$$
\begin{aligned}
& 1 \sigma^{*}:\left(\mathbf{0}, 1^{*}\right) \longmapsto \sum_{i_{1}=0}^{k_{1}-1}\left(-i_{1} \mathbf{e}_{d}, 1^{*}\right)+\sum_{i_{2}=0}^{k_{2}-1}\left(-i_{2} \mathbf{e}_{d}, 2^{*}\right) \\
&+\cdots+\sum_{i_{d-1}=0}^{k_{d-1}-1}\left(-i_{d-1} \mathbf{e}_{d}, d-1^{*}\right)+\left(\mathbf{0}, d^{*}\right), \\
&\left(\mathbf{0}, 2^{*}\right) \\
&\left(\mathbf{0}, 3^{*}\right) \\
& \stackrel{\longmapsto}{\longmapsto}\left(-k_{1} \mathbf{e}_{d}, 1^{*}\right), \\
&\left(\mathbf{0}, d^{*}\right) \stackrel{\left.\mathbf{e}_{d}, 2^{*}\right),}{\longmapsto}\left(-k_{d-1} \mathbf{e}_{d}, d-1^{*}\right) .
\end{aligned}
$$

Hence, $M^{-1} X_{i}(1 \leq i \leq d)$ are given by

$$
\begin{aligned}
M^{-1} X_{1} & =\lim _{n \rightarrow \infty} M^{n-1} \pi\left({ }^{1} \sigma^{*^{n-1}}\left({ }^{1} \sigma^{*}\left(\mathbf{0}, 1^{*}\right)\right)\right) \\
= & \lim _{n \rightarrow \infty} M^{n-1} \pi\left({ } ^ { 1 } \sigma ^ { * n - 1 } \left(\sum_{i_{1}=0}^{k_{1}-1}\left(-i_{1} \mathbf{e}_{d}, 1^{*}\right)+\cdots\right.\right. \\
& \left.\left.+\sum_{i_{d-1}=0}^{k_{d-1}-1}\left(-i_{d-1} \mathbf{e}_{d}, d-1^{*}\right)+\left(\mathbf{0}, d^{*}\right)\right)\right)
\end{aligned}
$$




$$
\begin{aligned}
&=\bigcup_{i_{1}=0}^{k_{1}-1}\left(X_{1}-i_{1} \pi \mathbf{e}_{d}\right) \cup \cdots \cup \bigcup_{i_{d-1}=0}^{k_{d-1}-1}\left(X_{d-1}-i_{d-1} \pi \mathbf{e}_{d}\right) \cup X_{d} \\
& M^{-1} X_{2}=\lim _{n \rightarrow \infty} M^{n-1} \pi\left({ }^{1} \sigma^{* n-1}\left({ }^{1} \sigma^{*}\left(\mathbf{0}, 2^{*}\right)\right)\right) \\
&=\lim _{n \rightarrow \infty} M^{n-1} \pi\left({ }^{1} \sigma^{* n-1}\left(-k_{1} \mathbf{e}_{d}, 1^{*}\right)\right) \\
&=X_{1}-k_{1} \pi \mathbf{e}_{d}, \\
& \vdots \\
& M^{-1} X_{d}=\lim _{n \rightarrow \infty} M^{n-1} \pi\left({ }^{1} \sigma^{* n-1}\left({ }^{1} \sigma^{*}\left(\mathbf{0}, d^{*}\right)\right)\right) \\
&=\lim _{n \rightarrow \infty} M^{n-1} \pi\left({ }^{1} \sigma^{* n-1}\left(-k_{d-1} \mathbf{e}_{d}, d-1^{*}\right)\right) \\
&=X_{d-1}-k_{d-1} \pi \mathbf{e}_{d} .
\end{aligned}
$$

Then applying $M$, from the property $M \pi \mathbf{e}_{d}=\pi M \mathbf{e}_{d}=\pi \mathbf{e}_{1}$, we have

$$
\left\{\begin{aligned}
X_{1} & =\bigcup_{i_{1}=0}^{k_{1}-1}\left(M X_{1}-i_{1} \pi \mathbf{e}_{1}\right) \cdots \bigcup_{i_{d-1}=0}^{k_{d-1}-1}\left(M X_{d-1}-i_{d-1} \pi \mathbf{e}_{1}\right) \cup M X_{d} \\
X_{2} & =M X_{1}-k_{1} \pi \mathbf{e}_{1} \\
& \vdots \\
X_{d} & =M X_{d-1}-k_{d-1} \pi \mathbf{e}_{1}
\end{aligned}\right.
$$

$$
\begin{aligned}
X & =\bigcup_{i=1}^{d} X_{i} \\
& =\bigcup_{i_{1}=0}^{k_{1}}\left(M X_{1}-i_{1} \pi \mathbf{e}_{1}\right) \cdots \bigcup_{i_{d-1}=0}^{k_{d-1}}\left(M X_{d-1}-i_{d-1} \pi \mathbf{e}_{1}\right) \cup M X_{d}
\end{aligned}
$$

Since $X_{i}$ are disjont up to a set of measure 0 , the partition of $X$ is constructed. By using the partition (3.3), the transformation $T_{\beta}^{*}$ on $X$ without boundaries can be defined as follows:

(3.4) $T_{\beta}^{*} \mathbf{x}=M^{-1} \mathbf{x}+b^{*} \pi \mathbf{e}_{d}$ if $\mathbf{x} \in M X_{j}-b^{*} \pi \mathbf{e}_{1}$ for some $j$ and $b^{*}$. 
Then for $\mathbf{x} \in X$ satisfying the condition that $T_{\beta}^{* k} x$ are not on the boundaries of $X^{i}$ for any $k$, there exists an infinite sequence $\left(b_{k}^{*}\right)_{k=1}^{\infty}$ such that

$$
T_{\beta}^{* k-1} \mathbf{x} \in M X_{j(k)}-b_{k}^{*} \pi \mathbf{e}_{1}
$$

and $\mathbf{x}$ is represented by

$$
\mathbf{x}=-\sum_{k=1}^{\infty} b_{k}^{*} M^{k-1} \pi \mathbf{e}_{1}
$$

Note that $(j(k))_{k=1}^{\infty}$ is the orbit of the point $\mathbf{x}$, that is,

$$
T_{\beta}^{* k} \mathbf{x} \in X_{j(k)}
$$

From the set equations (3.2) and (3.5) we can see that

$$
\begin{aligned}
T_{\beta}^{*}\left(X_{1}^{\circ}\right) & =X_{1}^{\circ} \cup X_{2}^{\circ} \cup \cdots \cup X_{d}^{\circ} \\
T_{\beta}^{*}\left(X_{2}^{\circ}\right) & =X_{1}^{\circ} \\
T_{\beta}^{*}\left(X_{3}^{\circ}\right) & =X_{2}^{\circ} \\
& \vdots \\
T_{\beta}^{*}\left(X_{d}^{\circ}\right) & =X_{d-1}^{\circ},
\end{aligned}
$$

where for each $i X_{i}^{\circ}$ is given by

$$
X_{i}^{\circ}=\left\{\begin{array}{l|l}
\mathbf{x} \in X_{i} & \begin{array}{l}
T_{\beta}^{* k} \mathbf{x} \text { are not on the boundaries of } X_{j} \text { for any } k \\
\text { and any } j
\end{array}
\end{array}\right\} .
$$

Hence, an infinite walk $\left(b_{k}^{*}\right)_{k=1}^{\infty}$ is obtained from the labeled graph $\mathcal{G}^{*}$, which is the dual graph of $\mathcal{G}$. Here, the dual graph $G^{*}$ is the graph with the same vertices as $G$, but with each edge in $G$ reversed in direction. We can deal with all points of $X_{i}$ successfully. As a consequence, we know that the domains $X_{i}$ s $(1 \leq i \leq d)$ are given by

$$
X_{i}=\left\{\begin{array}{l|l}
-\sum_{k=1}^{\infty} b_{k}^{*} M^{k-1} \pi \mathbf{e}_{1} & \begin{array}{l}
\left(b_{k}^{*}\right)_{k=1}^{\infty} \text { is an admissible walk starting } \\
\text { at } i \text { in } \mathcal{G}^{*}
\end{array}
\end{array}\right\}
$$

See details in [2] and [6]. 


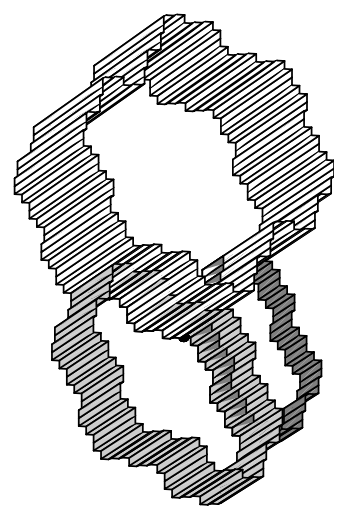

Figure 5: The figure of $\widehat{X}=\bigcup_{i=1}^{d} \widehat{X_{i}}$ in case $k_{1}=k_{2}=1$.

\section{§4. The natural extension of the $\beta$-transformation $T_{\beta}$}

Let for each $i(1 \leq i \leq d) \widehat{X_{i}} \subset \mathbb{R}^{d}$ be the following domain:

$$
\widehat{X}_{i}=\left\{t \boldsymbol{\alpha}+\mathbf{x} \mid 0 \leq t<\gamma_{i} \text { and } \mathbf{x} \in X_{i}\right\} \text {. }
$$

And we define $\widehat{X}$ by

$$
\widehat{X}=\bigcup_{i=1}^{d} \widehat{X_{i}}
$$

See Figure 5.

Let $\widehat{T_{\beta}}$ be the transformation on $\widehat{X}$ given by

$$
\widehat{T_{\beta}}(\underline{\underline{t}} \boldsymbol{\alpha}+\mathbf{x})=\frac{(\beta t-[\beta t])}{\frac{(\beta t}{T_{\beta} t}} \boldsymbol{\alpha}+M \mathbf{x}-[\beta t] \pi \mathbf{e}_{1} .
$$

Note that $\widehat{T_{\beta}}$ is just the $\beta$-transformation $T_{\beta}$ on the direction $\boldsymbol{\alpha}$. We know that for any $\mathbf{z}=t \boldsymbol{\alpha}+\mathbf{x} \in \widehat{X}$,

$$
\widehat{T_{\beta}}(\mathbf{z})=M \mathbf{z}-[\beta t] \mathbf{e}_{1}
$$

$\widehat{T_{\beta}}$ will be a toral automorphism associated with $M$ on the fundamental domain $\widehat{X}$.

From the partition (3.3) and the property (2.4) we have the following result. 
Proposition 4.1. $\widehat{T_{\beta}}$ is surjective and injective on $\widehat{X}$ except on the boundary.

Proof. For any $\mathbf{y} \in \widehat{X}$ there exist $\mathbf{y}^{\prime} \in X_{i}(1 \leq i \leq d)$ and $t^{\prime}\left(0 \leq t^{\prime}<\right.$ $\left.\gamma_{i} \leq 1\right)$ such that

$$
\mathbf{y}=t^{\prime} \boldsymbol{\alpha}+\mathbf{y}^{\prime}
$$

From the partition (3.2), we have

$$
\mathbf{y}=t^{\prime} \boldsymbol{\alpha}+M \mathbf{x}^{\prime}-k \pi \mathbf{e}_{1} \quad \text { for some } \mathbf{x}^{\prime} \in X_{j} \text { and } 0 \leq k \leq k_{1} .
$$

Let

$$
\mathbf{x}=\left(\frac{k}{\beta}+\frac{t^{\prime}}{\beta}\right) \boldsymbol{\alpha}+\mathbf{x}^{\prime}
$$

Then

$$
\widehat{T_{\beta}} \mathbf{x}=t^{\prime} \boldsymbol{\alpha}+M \mathbf{x}^{\prime}-k \pi \mathbf{e}_{1}=\mathbf{y} .
$$

If $i=1,0 \leq t^{\prime}<1$ and $k=0,1, \ldots, k_{j}-1$. Here we set $k_{d}=1$. Then

$$
0 \leq \frac{k}{\beta}+\frac{t^{\prime}}{\beta}<\frac{k_{j}-1}{\beta}+\frac{1}{\beta}=\frac{k_{j}}{\beta}=. k_{j} \leq \gamma_{j} \text {. }
$$

If $i=2, \ldots, d$, we know that $0 \leq t^{\prime}<\gamma_{i}=. k_{i} \ldots k_{d-1} 1, j=i-1$, and $k=k_{i-1}$. Hence

$$
0 \leq \frac{k}{\beta}+\frac{t^{\prime}}{\beta}<\frac{k_{i-1}}{\beta}+\frac{\gamma_{i}}{\beta}=. k_{i-1} \ldots k_{d-1} 1=\gamma_{i-1}=\gamma_{j}
$$

Therefore for any $i$, we see that $\mathbf{x} \in \widehat{X_{j}} \subset \widehat{X}$. Hence $\widehat{T_{\beta}}$ is surjective. And except for the boundary, $i, t^{\prime}, j$, and $k$ are uniquely determined by $\mathbf{y}$. Therefore $\widehat{T_{\beta}}$ is almost everywhere injective.

Therefore $\widehat{T_{\beta}}$ is the natural extension of the transformation $T_{\beta}$.

Recall that the domain $X$ is on the plane $\mathcal{P}$, which is orthogonal to $\gamma$. We put

$$
\begin{aligned}
Q & :=\left[\begin{array}{cccccccc}
\alpha_{1}^{(1)} & \ldots & \alpha_{1}^{\left(r_{1}\right)} & \Re \alpha_{1}^{\left(r_{1}+1\right)} & -\Im \alpha_{1}^{\left(r_{1}+1\right)} & \ldots & \Re \alpha_{1}^{\left(r_{1}+r_{2}\right)} & -\Im \alpha_{1}^{\left(r_{1}+r_{2}\right)} \\
\alpha_{2}^{(1)} & \ldots & \alpha_{2}^{\left(r_{1}\right)} & \Re \alpha_{2}^{\left(r_{1}+1\right)} & -\Im \alpha_{2}^{\left(r_{1}+1\right)} & \ldots & \Re \alpha_{2}^{\left(r_{1}+r_{2}\right)} & -\Im \alpha_{2}^{\left(r_{1}+r_{2}\right)} \\
\vdots & & \vdots & \vdots & \vdots & & \vdots & \vdots \\
\alpha_{d}^{(1)} & \ldots & \alpha_{d}^{\left(r_{1}\right)} & \Re \alpha_{d}^{\left(r_{1}+1\right)} & -\Im \alpha_{d}^{\left(r_{1}+1\right)} & \ldots & \Re \alpha_{d}^{\left(r_{1}+r_{2}\right)} & -\Im \alpha_{d}^{\left(r_{1}+r_{2}\right)}
\end{array}\right] \\
= & :\left[\begin{array}{lllll}
\boldsymbol{\alpha}, \boldsymbol{\alpha}_{2}, \ldots, \boldsymbol{\alpha}_{d}
\end{array}\right],
\end{aligned}
$$


where $\Re$ indicates the real part and $\Im$ indicates the imaginary part. The plane $\mathcal{P}$ is spanned by $\boldsymbol{\alpha}_{2}, \boldsymbol{\alpha}_{3}, \ldots$, and $\boldsymbol{\alpha}_{d}$, because $\boldsymbol{\alpha}_{i}(2 \leq i \leq d)$ and $\boldsymbol{\gamma}$ intersect orthogonally and $\boldsymbol{\alpha}_{i}$ s are linearly independent.

Let us define the domains $\widehat{Y}$ and $\widehat{Y}_{i}(1 \leq i \leq d)$ as follows:

$$
\widehat{Y}:=Q^{-1}(\widehat{X}) \text { and } \widehat{Y}_{i}:=Q^{-1}\left(\widehat{X}_{i}\right)
$$

We will make preparations for the explicit representation of $\widehat{Y}_{i}$.

Define a $d \times d$ matrix

$$
\begin{aligned}
& P:=\left[\begin{array}{cccccccc}
\alpha_{1}^{(1)} & \cdots & \alpha_{1}^{\left(r_{1}\right)} & \alpha_{1}^{\left(r_{1}+1\right)} & \overline{\alpha_{1}^{\left(r_{1}+1\right)}} & \cdots & \alpha_{1}^{\left(r_{1}+r_{2}\right)} & \overline{\alpha_{1}^{\left(r_{1}+r_{2}\right)}} \\
\alpha_{2}^{(1)} & \cdots & \alpha_{2}^{\left(r_{1}\right)} & \alpha_{2}^{\left(r_{1}+1\right)} & \overline{\alpha_{2}^{\left(r_{1}+1\right)}} & \cdots & \alpha_{2}^{\left(r_{1}+r_{2}\right)} & \overline{\alpha_{2}^{\left(r_{1}+r_{2}\right)}} \\
\vdots & & \vdots & \vdots & \vdots & & \vdots & \vdots \\
\alpha_{d}^{(1)} & \cdots & \alpha_{d}^{\left(r_{1}\right)} & \alpha_{d}^{\left(r_{1}+1\right)} & \overline{\alpha_{d}^{\left(r_{1}+1\right)}} & \cdots & \alpha_{d}^{\left(r_{1}+r_{2}\right)} & \overline{\alpha_{d}^{\left(r_{1}+r_{2}\right)}}
\end{array}\right] \\
& =:\left[\boldsymbol{\alpha}, \mathbf{u}_{2}, \ldots, \mathbf{u}_{d}\right] \text {. }
\end{aligned}
$$

Let

$$
U:=I_{r_{1}} \oplus\left[\begin{array}{cc}
1 & 1 \\
-\sqrt{-1} & \sqrt{-1}
\end{array}\right] \oplus\left[\begin{array}{cc}
1 & 1 \\
-\sqrt{-1} & \sqrt{-1}
\end{array}\right] \oplus \cdots \oplus\left[\begin{array}{cc}
1 & 1 \\
-\sqrt{-1} & \sqrt{-1}
\end{array}\right],
$$

where $A \oplus B$ is a matrix of a form:

$$
\left[\begin{array}{ll}
A & \mathbf{0} \\
\mathbf{0} & B
\end{array}\right]
$$

and $I_{r_{1}}$ is the identity matrix of size $r_{1}$. Then

$$
Q U=P
$$

From $I_{d}=P \cdot P^{-1}$, we have

$$
\mathbf{e}_{1}=\left(P^{-1}\right)_{11} \boldsymbol{\alpha}+\left(P^{-1}\right)_{21} \mathbf{u}_{2}+\cdots+\left(P^{-1}\right)_{d 1} \mathbf{u}_{d}
$$

Each $\mathbf{u}_{i}(2 \leq i \leq d)$ is also orthogonal to $\gamma$. Therefore,

$$
\left\langle\mathbf{e}_{1}, \gamma\right\rangle=\left\langle\left(P^{-1}\right)_{11} \boldsymbol{\alpha}, \boldsymbol{\gamma}\right\rangle=\left(P^{-1}\right)_{11}\langle\boldsymbol{\alpha}, \boldsymbol{\gamma}\rangle=\left(P^{-1}\right)_{11}
$$

It follows that

$$
\left(P^{-1}\right)_{11}=1
$$


and

$$
P^{-1} \mathbf{e}_{1}={ }^{t}[1,1, \ldots, 1] .
$$

You can see the detailed proof in [11]. According to the relation $Q U=P$

$$
Q^{-1} \mathbf{e}_{1}={ }^{t}[\underbrace{1,1, \ldots, 1}_{r_{1}}, \underbrace{2,0, \ldots, 2,0}_{2 r_{2}}] .
$$

Moreover, from $I_{d}=Q \cdot Q^{-1}$

$$
\mathbf{e}_{1}=\boldsymbol{\alpha}+\boldsymbol{\alpha}_{2}+\cdots+\boldsymbol{\alpha}_{r_{1}}+2 \boldsymbol{\alpha}_{r_{1}+1}+2 \boldsymbol{\alpha}_{r_{1}+3}+\cdots+2 \boldsymbol{\alpha}_{d-1} .
$$

Since $\pi$ is the projection along $\boldsymbol{\alpha}$,

$$
\pi \mathbf{e}_{1}=\boldsymbol{\alpha}_{2}+\cdots+\boldsymbol{\alpha}_{r_{1}}+2 \boldsymbol{\alpha}_{r_{1}+1}+2 \boldsymbol{\alpha}_{r_{1}+3}+\cdots+2 \boldsymbol{\alpha}_{d-1} .
$$

Hence

$$
Q^{-1} \pi \mathbf{e}_{1}={ }^{t}[\underbrace{0,1,1, \ldots, 1}_{r_{1}}, \underbrace{2,0, \ldots, 2,0}_{2 r_{2}}] .
$$

LEMMA 4.2. The following relation holds:

$$
\begin{aligned}
M Q=Q & {\left[\begin{array}{llll}
\beta & & & \\
& \beta^{(2)} & & \\
& \ddots & \\
& & \beta^{\left(r_{1}\right)}
\end{array}\right] } \\
& \oplus\left[\begin{array}{cc}
\Re \beta^{\left(r_{1}+1\right)} & -\Im \beta^{\left(r_{1}+1\right)} \\
\Im \beta^{\left(r_{1}+1\right)} & \Re \beta^{\left(r_{1}+1\right)}
\end{array}\right] \oplus \cdots \oplus\left[\begin{array}{cc}
\Re \beta^{\left(r_{1}+r_{2}\right)} & -\Im \beta^{\left(r_{1}+r_{2}\right)} \\
\Im \beta^{\left(r_{1}+r_{2}\right)} & \Re \beta^{\left(r_{1}+r_{2}\right)}
\end{array}\right] .
\end{aligned}
$$

Proof. The relation (2.2) implies that

$$
M P=P D,
$$

where $D$ is the diagonal matrix

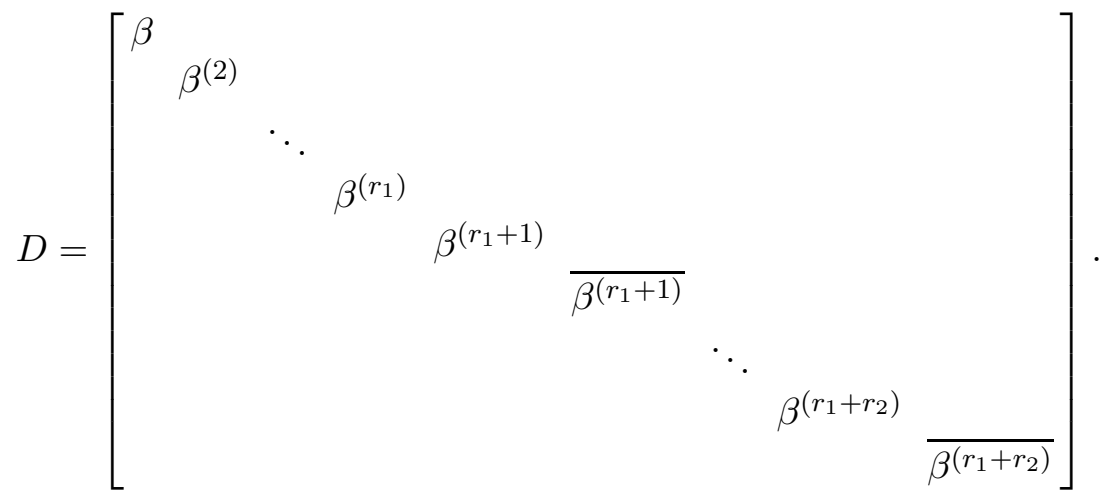


Then, from the relation $P=Q U$,

$$
M Q U=Q U D
$$

Using

$$
U^{-1}=I_{r_{1}} \oplus \frac{1}{2}\left[\begin{array}{cc}
1 & \sqrt{-1} \\
1 & -\sqrt{-1}
\end{array}\right] \oplus \cdots \oplus \frac{1}{2}\left[\begin{array}{cc}
1 & \sqrt{-1} \\
1 & -\sqrt{-1}
\end{array}\right],
$$

we have

$$
\begin{aligned}
& Q^{-1} M Q=U D U^{-1} \\
& =\left[\begin{array}{llll}
\beta & & & \\
& \beta^{(2)} & & \\
& & \ddots & \\
& & & \beta^{\left(r_{1}\right)}
\end{array}\right] \\
& \oplus\left[\begin{array}{cc}
\Re \beta^{\left(r_{1}+1\right)} & -\Im \beta^{\left(r_{1}+1\right)} \\
\Im \beta^{\left(r_{1}+1\right)} & \Re \beta^{\left(r_{1}+1\right)}
\end{array}\right] \oplus \cdots \oplus\left[\begin{array}{cc}
\Re \beta^{\left(r_{1}+r_{2}\right)} & -\Im \beta^{\left(r_{1}+r_{2}\right)} \\
\Im \beta^{\left(r_{1}+r_{2}\right)} & \Re \beta^{\left(r_{1}+r_{2}\right)}
\end{array}\right] .
\end{aligned}
$$

Hereafter we represent $\widehat{Y}_{i}$ s as the domains in $\mathbb{R} \times \mathbb{R}^{d-1}$.

Proposition 4.3. The domains $\widehat{Y}_{i}$ s are given by

$$
\begin{aligned}
& \widehat{Y}_{i}=\left\{\left(t,-\sum_{k=1}^{\infty} b_{k}^{*} R^{k-1} \mathbf{v}\right) \mid\right. 0 \leq t<\gamma_{i} \text { and } \\
&\left.\left(b_{k}^{*}\right)_{k=1}^{\infty} \text { is an admissible walk starting at } i \text { in } \mathcal{G}^{*}\right\},
\end{aligned}
$$

where

$$
\begin{aligned}
& R=\left[\begin{array}{lll}
\beta^{(2)} & & \\
& \ddots & \\
& & \beta^{\left(r_{1}\right)}
\end{array}\right] \\
& \oplus\left[\begin{array}{cc}
\Re \beta^{\left(r_{1}+1\right)} & -\Im \beta^{\left(r_{1}+1\right)} \\
\Im \beta^{\left(r_{1}+1\right)} & \Re \beta^{\left(r_{1}+1\right)}
\end{array}\right] \oplus \cdots \oplus\left[\begin{array}{cc}
\Re \beta^{\left(r_{1}+r_{2}\right)} & -\Im \beta^{\left(r_{1}+r_{2}\right)} \\
\Im \beta^{\left(r_{1}+r_{2}\right)} & \Re \beta^{\left(r_{1}+r_{2}\right)}
\end{array}\right]
\end{aligned}
$$

and

$$
\mathbf{v}={ }^{t}[\underbrace{1, \ldots, 1}_{r_{1}-1}, \underbrace{2,0, \ldots, 2,0}_{2 r_{2}}]
$$


Proof. The definitions of $\widehat{Y}_{i}, \widehat{X}_{i}$, and $X_{i}$, that is, (4.3), (4.1), and (3.6), show that

$$
\begin{aligned}
\widehat{Y}_{i} & =Q^{-1}\left(\widehat{X_{i}}\right) \\
& =Q^{-1}\left\{t \boldsymbol{\alpha}-\sum_{k=1}^{\infty} b_{k}^{*} M^{k-1} \pi \mathbf{e}_{1} \mid(*) \text {-condition }\right\} \\
& =\left\{t Q^{-1} \boldsymbol{\alpha}-\sum_{k=1}^{\infty} b_{k}^{*} Q^{-1} M^{k-1} \pi \mathbf{e}_{1} \mid(*) \text {-condition }\right\} .
\end{aligned}
$$

By Lemma 4.2

$$
Q^{-1} M^{k-1}=\left(\beta^{k-1} \oplus R^{k-1}\right) Q^{-1}
$$

And using (4.4), we have

$$
\begin{aligned}
\widehat{Y}_{i} & =\left\{t \mathbf{e}_{1}-\sum_{k=1}^{\infty} b_{k}^{*}\left(\beta^{k-1} \oplus R^{k-1}\right) Q^{-1} \pi \mathbf{e}_{1} \mid(*) \text {-condition }\right\} \\
& =\left\{\left(t,-\sum_{k=1}^{\infty} b_{k}^{*} R^{k-1} \mathbf{v}\right) \mid(*) \text {-condition }\right\} .
\end{aligned}
$$

Here $(*)$-condition means that $0 \leq t<\gamma_{i}$ and $\left(b_{k}^{*}\right)_{k=1}^{\infty}$ is an admissible walk starting at $i$ in $\mathcal{G}^{*}$. Therefore we arrive at the conclusion of the assertion.

Naturally, we can define a transformation $\widehat{S_{\beta}}$ on $\widehat{Y}$ as follows:

$$
\widehat{S_{\beta}}:=Q^{-1} \circ \widehat{T_{\beta}} \circ Q
$$

Then $\widehat{S_{\beta}}$ is also a natural extension of $T_{\beta}$.

Proposition 4.4. The transformation $\widehat{S_{\beta}}$ on $\widehat{Y}$ is given by

$$
\widehat{S_{\beta}}(t, \mathbf{x})=(\beta t-[\beta t], R \mathbf{x}-[\beta t] \mathbf{v})
$$

and surjective. 
Proof. From (4.5) and (4.2), which are definitions of $\widehat{S_{\beta}}$ and $\widehat{T_{\beta}}$,

$$
\begin{aligned}
\widehat{S_{\beta}}(t, \mathbf{x}) & :=Q^{-1} \circ \widehat{T_{\beta}} \circ Q\left(t \mathbf{e}_{1}+0 \oplus \mathbf{x}\right) \\
& =Q^{-1} \circ \widehat{T_{\beta}}(t \boldsymbol{\alpha}+Q(0 \oplus \mathbf{x})) \\
& =Q^{-1}\left((\beta t-[\beta t]) \boldsymbol{\alpha}+M Q(0 \oplus \mathbf{x})-[\beta t] \pi \mathbf{e}_{1}\right) \\
& =(\beta t-[\beta t], R \mathbf{x}-[\beta t] \mathbf{v}) .
\end{aligned}
$$

Surjectivity of $\widehat{S_{\beta}}$ is obtained by Proposition 4.1 .

\section{$\S 5$. The reduction theorem}

In this section, we introduce reduced numbers and show our main theorem.

Let $\tilde{Y}\left(\subset \mathbb{R} \times \mathbb{R}^{d-1}\right)$ be the following product space:

$$
\widetilde{Y}:=[0,1) \times \mathbb{R}^{d-1} .
$$

Let $\widetilde{S_{\beta}}$ be the transformation on $\widetilde{Y}$ defined by

$$
\widetilde{S_{\beta}}(x, \mathbf{x}):=(\beta x-[\beta x], R \mathbf{x}-[\beta x] \mathbf{v}), \quad x \in[0,1) .
$$

Then the restriction of $\widetilde{S_{\beta}}$ on $\widehat{Y}(\subset \widetilde{Y})$ is $\widehat{S_{\beta}}$.

Define a map $\rho: \mathbb{Q}(\beta) \rightarrow \mathbb{R} \times \mathbb{R}^{d-1}$ by

$$
\rho(x)=\left(x,\left[\begin{array}{c}
x^{(2)} \\
\vdots \\
x^{\left(r_{1}\right)} \\
2 \Re x^{\left(r_{1}+1\right)} \\
2 \Im x^{\left(r_{1}+1\right)} \\
\vdots \\
2 \Re x^{\left(r_{1}+r_{2}\right)} \\
2 \Im x^{\left(r_{1}+r_{2}\right)}
\end{array}\right]\right) .
$$

$\widehat{Y}$. Definition 5.1. A real number $x \in \mathbb{Q}(\beta) \cap[0,1)$ is reduced if $\rho(x) \in$

In order to prove the main theorem, we will need some important lemmas. 
Lemma 5.1. Let $x \in \mathbb{Q}(\beta) \cap[0,1)$. Then

$$
\widetilde{S_{\beta}}(\rho(x))=\rho\left(T_{\beta} x\right) .
$$

Proof. From the definitions of $\widetilde{S_{\beta}}, \rho$, and $T_{\beta}$, we have

$$
\begin{aligned}
& \widetilde{S_{\beta}}(\rho(x))=\widetilde{S_{\beta}}\left(x,\left[\begin{array}{c}
x^{(2)} \\
\vdots \\
x^{\left(r_{1}\right)} \\
2 \Re x^{\left(r_{1}+1\right)} \\
2 \Im x^{\left(r_{1}+1\right)} \\
\vdots \\
2 \Re x^{\left(r_{1}+r_{2}\right)} \\
2 \Im x^{\left(r_{1}+r_{2}\right)}
\end{array}\right]\right) \\
& =\left[\begin{array}{c}
\beta^{(2)} x^{(2)} \\
\vdots \\
\beta^{\left(r_{1}\right)} x^{\left(r_{1}\right)} \\
2\left(\Re \beta^{\left(r_{1}+1\right)} \cdot \Re x^{\left(r_{1}+1\right)}-\Im \beta^{\left(r_{1}+1\right)} \cdot \Im x^{\left(r_{1}+1\right)}\right) \\
2\left(\Im \beta^{\left(r_{1}+1\right)} \cdot \Re x^{\left(r_{1}+1\right)}+\Re \beta^{\left(r_{1}+1\right)} \cdot \Im x^{\left(r_{1}+1\right)}\right) \\
\vdots \\
2\left(\Re \beta^{\left(r_{1}+r_{2}\right)} \cdot \Re x^{\left(r_{1}+r_{2}\right)}-\Im \beta^{\left(r_{1}+r_{2}\right)} \cdot \Im x^{\left(r_{1}+r_{2}\right)}\right) \\
2\left(\Im \beta^{\left(r_{1}+r_{2}\right)} \cdot \Re x^{\left(r_{1}+r_{2}\right)}+\Re \beta^{\left(r_{1}+r_{2}\right)} \cdot \Im x^{\left(r_{1}+r_{2}\right)}\right)
\end{array}\right] \\
& \left.-[\beta x]\left[\begin{array}{c}
1 \\
\vdots \\
1 \\
2 \\
0 \\
\vdots \\
2 \\
0
\end{array}\right]\right)
\end{aligned}
$$


using the relations $\Re(x y)=\Re x \Re y-\Im x \Im y$ and $\Im(x y)=\Re x \Im y+\Im x \Re y$,

$$
\begin{aligned}
& =\left(\beta x-[\beta x],\left[\begin{array}{c}
(\beta x)^{(2)}-[\beta x] \\
\vdots \\
(\beta x)^{\left(r_{1}\right)}-[\beta x] \\
2 \Re\left((\beta x)^{\left(r_{1}+1\right)}-[\beta x]\right) \\
2 \Im\left((\beta x)^{\left(r_{1}+1\right)}-[\beta x]\right) \\
\vdots \\
2 \Re\left((\beta x)^{\left(r_{1}+r_{2}\right)}-[\beta x]\right) \\
2 \Im\left((\beta x)^{\left(r_{1}+r_{2}\right)}-[\beta x]\right)
\end{array}\right]\right) \\
& =\rho(\beta x-[\beta x]) \\
& =\rho\left(T_{\beta} x\right) .
\end{aligned}
$$

Therefore we arrive at the conclusion.

Lemma 5.2. Let $x \in \mathbb{Q}(\beta) \cap[0,1)$ be reduced. Then

(1) $T_{\beta} x$ is reduced,

(2) there exists $x^{*}$ such that $x^{*}$ is reduced and $T_{\beta} x^{*}=x$.

Proof. Since $x \in \mathbb{Q}(\beta) \cap[0,1)$ is reduced, $\rho(x) \in \widehat{Y}$.

(1) From Lemma 5.1,

$$
\widehat{S_{\beta}}(\rho(x))=\rho\left(T_{\beta} x\right) \in \widehat{Y}
$$

Hence $T_{\beta} x$ is reduced.

(2) From Proposition $4.4, \widehat{S_{\beta}}$ is surjective on $\widehat{Y}$. Thus there exist $\left(x^{*}, \mathbf{x}\right) \in \widehat{Y}$ such that

$$
\widehat{S_{\beta}}\left(x^{*}, \mathbf{x}\right)=\rho(x) .
$$

Comparing first coordinates in both sides, we see that

$$
T_{\beta} x^{*}=x
$$

To verify $x^{*}$ is reduced, we will only show

$$
\left(x^{*}, \mathbf{x}\right)=\rho\left(x^{*}\right) .
$$

Then $\rho\left(x^{*}\right) \in \widehat{Y}$ implies that $x^{*}$ is reduced.

We put

$$
\mathbf{x}={ }^{t}\left[x_{2}, \ldots, x_{r_{1}}, x_{r_{1}+1}, \widetilde{x_{r_{1}+1}}, \ldots, x_{r_{1}+r_{2}}, \widetilde{x_{r_{1}+r_{2}}}\right]
$$


Then (5.1) shows that

$$
\beta x^{*}-\left[\beta x^{*}\right]=x
$$

and

$$
R \mathbf{x}-\left[\beta x^{*}\right] \mathbf{v}=\left[\begin{array}{c}
x^{(2)} \\
\vdots \\
x^{\left(r_{1}\right)} \\
2 \Re x^{\left(r_{1}+1\right)} \\
2 \Im x^{\left(r_{1}+1\right)} \\
\vdots \\
2 \Re x^{\left(r_{1}+r_{2}\right)} \\
2 \Im x^{\left(r_{1}+r_{2}\right)}
\end{array}\right]
$$

So that,

$$
\begin{aligned}
& {\left[\begin{array}{c}
\beta^{(2)} x_{2}-\left[\beta x^{*}\right] \\
\vdots \\
\beta^{\left(r_{1}\right)} x_{r_{1}}-\left[\beta x^{*}\right] \\
\Re \beta^{\left(r_{1}+1\right)} \cdot x_{r_{1}+1}-\Im \beta^{\left(r_{1}+1\right)} \cdot \widehat{x_{r_{1}+1}}-2\left[\beta x^{*}\right] \\
\Im \beta^{\left(r_{1}+1\right)} \cdot x_{r_{1}+1}+\Re \beta^{\left(r_{1}+1\right)} \cdot \widehat{x_{r_{1}+1}} \\
\vdots \\
\Re \beta^{\left(r_{1}+r_{2}\right)} \cdot x_{r_{1}+r_{2}}-\Im \beta^{\left(r_{1}+r_{2}\right)} \cdot \widehat{x_{r_{1}+r_{2}}}-2\left[\beta x^{*}\right] \\
\Im \beta^{\left(r_{1}+r_{2}\right)} \cdot x_{r_{1}+r_{2}}+\Re \beta^{\left(r_{1}+r_{2}\right)} \cdot \widehat{x_{r_{1}+r_{2}}}
\end{array}\right]} \\
& =\left[\begin{array}{c}
\beta^{(2)} x^{*(2)}-\left[\beta x^{*}\right] \\
\vdots \\
\beta^{\left(r_{1}\right)} x^{*\left(r_{1}\right)}-\left[\beta x^{*}\right] \\
2 \Re\left(\beta^{\left(r_{1}+1\right)} x^{*\left(r_{1}+1\right)}-\left[\beta x^{*}\right]\right) \\
2 \Im\left(\beta^{\left(r_{1}+1\right)} x^{*\left(r_{1}+1\right)}-\left[\beta x^{*}\right]\right) \\
\vdots \\
2 \Re\left(\beta^{\left(r_{1}+r_{2}\right)} x^{*\left(r_{1}+r_{2}\right)}-\left[\beta x^{*}\right]\right) \\
2 \Im\left(\beta^{\left(r_{1}+r_{2}\right)} x^{*\left(r_{1}+r_{2}\right)}-\left[\beta x^{*}\right]\right)
\end{array}\right] .
\end{aligned}
$$

Thus

$$
x_{2}=x^{*(2)}, \ldots, x_{r_{1}}=x^{*\left(r_{1}\right)},
$$


and for $1 \leq j \leq r_{2}$

$$
\begin{aligned}
& \Re \beta^{\left(r_{1}+j\right)} \cdot x_{r_{1}+j}-\Im \beta^{\left(r_{1}+j\right)} \cdot \widetilde{x_{r_{1}+j}} \\
& \quad=2 \Re\left(\beta^{\left(r_{1}+j\right)} x^{*\left(r_{1}+j\right)}\right) \\
& \quad=2 \Re \beta^{\left(r_{1}+j\right)} \Re x^{*\left(r_{1}+j\right)}-2 \Im \beta^{\left(r_{1}+j\right)} \Im x^{*\left(r_{1}+j\right)}, \\
& \Im \beta^{\left(r_{1}+j\right)} \cdot x_{r_{1}+j}+\Re \beta^{\left(r_{1}+j\right)} \cdot \widetilde{x_{r_{1}+j}} \\
& \quad=2 \Im\left(\beta^{\left(r_{1}+j\right)} x^{*\left(r_{1}+j\right)}\right) \\
& \quad=2 \Re \beta^{\left(r_{1}+j\right)} \Im x^{*\left(r_{1}+j\right)}+2 \Im \beta^{\left(r_{1}+j\right)} \Re x^{*\left(r_{1}+j\right)} .
\end{aligned}
$$

Then for $1 \leq j \leq r_{2}$, we have

$$
\begin{aligned}
& \left(x_{r_{1}+j}-2 \Re x^{*\left(r_{1}+j\right)}\right) \Re \beta^{\left(r_{1}+j\right)}-\left(\widetilde{x_{r_{1}+j}}-2 \Im x^{*\left(r_{1}+j\right)}\right) \Im \beta^{\left(r_{1}+j\right)}=0, \\
& \left(x_{r_{1}+j}-2 \Re x^{*\left(r_{1}+j\right)}\right) \Im \beta^{\left(r_{1}+j\right)}+\left(\widetilde{x_{r_{1}+j}}-2 \Im x^{*\left(r_{1}+j\right)}\right) \Re \beta^{\left(r_{1}+j\right)}=0 .
\end{aligned}
$$

Thus

$$
x_{r_{1}+j}=2 \Re x^{*\left(r_{1}+j\right)} \text { and } \widetilde{x_{r_{1}+j}}=2 \Im x^{*\left(r_{1}+j\right)} .
$$

Therefore

$$
\left(x^{*}, \mathbf{x}\right)=\rho\left(x^{*}\right) .
$$

Thus we obtain the assertion (2).

By the lemmas above, we can get a sufficient condition for pure periodicity of $\beta$-expansions.

Proposition 5.3. Let $x \in \mathbb{Q}(\beta) \cap[0,1)$ be reduced. Then $x$ has a purely periodic $\beta$-expansion.

Proof. Lemma 5.2 (2) shows that there exist $x_{i}^{*} \mathrm{~s}$ such that $x_{i}^{*} \mathrm{~s}$ are reduced and $T_{\beta} x_{i}^{*}=x_{i-1}^{*}$, where we set $x_{0}^{*}=x$. Here, we put

$$
x=\frac{p_{0}}{q} \text { for some } q \in \mathbb{Z}, p_{0} \in \mathbb{Z}[\beta] .
$$

Then $T_{\beta} x_{1}^{*}=x$ implies that

$$
\beta x_{1}^{*}-\left[\beta x_{1}^{*}\right]=x .
$$


So that

$$
x_{1}^{*}=\frac{\left[\beta x_{1}^{*}\right]}{\beta}+\frac{x}{\beta}=\frac{p_{1}}{q} \text { for some } p_{1} \in \mathbb{Z}[\beta] .
$$

Inductively we can see for every $k$

$$
x_{k}^{*}=\frac{p_{k}}{q} \text { for some } p_{k} \in \mathbb{Z}[\beta] .
$$

Let $b_{j}$ be positive real numbers. Only in this proof, we denote by $x^{(j)}$ $(1 \leq j \leq d)$ algebraic conjugates of $x$ and $x^{(1)}=x$. Let

$$
C=\left\{x \in \mathbb{Z}[\beta]|| x^{(j)} \mid \leq b_{j}\right\}
$$

Obviously, $C$ is a finite set. As $\widehat{Y}$ is bounded, we can see the set $\left\{x_{i}^{*}\right\}_{i=0}^{\infty}$ is a finite set. Hence there exist $j$ and $k(j>k)$ such that

$$
x_{j}^{*}=x_{j-k}^{*}
$$

Applying $T_{\beta}^{j-k}$ we get

$$
x_{k}^{*}=x .
$$

Hence

$$
T_{\beta}^{k} x=x
$$

Therefore $x$ has a purely periodic $\beta$-expansion.

Lemma 5.2 and Proposition 5.3 show that the transformation $T_{\beta}$ restricted to $\mathbb{Q}(\beta) \cap[0,1)$ is bijective.

To complete the proof of our main theorem, the following proposition is positively necessary.

Proposition 5.4. Let $x \in \mathbb{Q}(\beta) \cap[0,1)$. Then there exists $N_{1}>0$ such that $T_{\beta}^{N} x$ are reduced for any $N \geq N_{1}$.

Proof. Consider the Euclidean distance $d$ between ${\widetilde{S_{\beta}}}^{k}(\rho(x))$ and $\widetilde{S}_{\beta}^{k}(x, \mathbf{0})$ for $k \geq 0$. Since the first coordinates of these points are equal, 
this coincides with the distance between the origin of $\mathbb{R}^{d-1}$ and

$$
R^{k}\left[\begin{array}{c}
x^{(2)} \\
\vdots \\
x^{\left(r_{1}\right)} \\
2 \Re x^{\left(r_{1}+1\right)} \\
2 \Im x^{\left(r_{1}+1\right)} \\
\vdots \\
2 \Re x^{\left(r_{1}+r_{2}\right)} \\
2 \Im x^{\left(r_{1}+r_{2}\right)}
\end{array}\right] .
$$

By $s(x)$ we denote this distance. As $R^{k}$ s are given by

$$
\begin{aligned}
& R^{k}=\left[\begin{array}{lll}
\left(\beta^{(2)}\right)^{k} & & \\
& \ddots & \\
& & \left(\beta^{\left(r_{1}\right)}\right)^{k}
\end{array}\right] \\
& \oplus\left|\beta^{\left(r_{1}+1\right)}\right|^{2 k}\left[\begin{array}{ll}
\Re \beta^{\left(r_{1}+1\right)} /\left|\beta^{\left(r_{1}+1\right)}\right|^{2} & -\Im \beta^{\left(r_{1}+1\right)} /\left|\beta^{\left(r_{1}+1\right)}\right|^{2} \\
\Im \beta^{\left(r_{1}+1\right)} /\left|\beta^{\left(r_{1}+1\right)}\right|^{2} & \Re \beta^{\left(r_{1}+1\right)} /\left|\beta^{\left(r_{1}+1\right)}\right|^{2}
\end{array}\right]^{k} \\
& \oplus \cdots \oplus\left|\beta^{\left(r_{1}+r_{2}\right)}\right|^{2 k}\left[\begin{array}{ll}
\Re \beta^{\left(r_{1}+r_{2}\right)} /\left|\beta^{\left(r_{1}+r_{2}\right)}\right|^{2} & -\Im \beta^{\left(r_{1}+r_{2}\right)} /\left|\beta^{\left(r_{1}+r_{2}\right)}\right|^{2} \\
\Im \beta^{\left(r_{1}+r_{2}\right)} /\left|\beta^{\left(r_{1}+r_{2}\right)}\right|^{2} & \Re \beta^{\left(r_{1}+r_{2}\right)} /\left|\beta^{\left(r_{1}+r_{2}\right)}\right|^{2}
\end{array}\right]^{k},
\end{aligned}
$$

we have

$$
\begin{aligned}
s(x)^{2}= & \left(\beta^{(2)}\right)^{2 k}\left(x^{(2)}\right)^{2}+\cdots+\left(\beta^{\left(r_{1}\right)}\right)^{2 k}\left(x^{\left(r_{1}\right)}\right)^{2} \\
& +\left|\beta^{\left(r_{1}+1\right)}\right|^{2 k}\left\{\left(2 \Re x^{\left(r_{1}+1\right)}\right)^{2}+\left(2 \Im x^{\left(r_{1}+1\right)}\right)^{2}\right\} \\
& +\cdots+\left|\beta^{\left(r_{1}+r_{2}\right)}\right|^{2 k}\left\{\left(2 \Re x^{\left(r_{1}+r_{2}\right)}\right)^{2}+\left(2 \Im x^{\left(r_{1}+r_{2}\right)}\right)^{2}\right\} .
\end{aligned}
$$

If we put

$$
u=\max \left\{\left|\beta^{(2)}\right|, \ldots,\left|\beta^{\left(r_{1}\right)}\right|,\left|\beta^{\left(r_{1}+1\right)}\right|, \ldots,\left|\beta^{\left(r_{1}+r_{2}\right)}\right|\right\},
$$

then $0<u<1$ and

$$
\begin{aligned}
s(x) \leq u^{k} \cdot\left\{\left(x^{(2)}\right)^{2}+\cdots+\right. & \left(x^{\left(r_{1}\right)}\right)^{2}+\left(2 \Re x^{\left(r_{1}+1\right)}\right)^{2}+\left(2 \Im x^{\left(r_{1}+1\right)}\right)^{2} \\
& \left.+\cdots+\left(2 \Re x^{\left(r_{1}+r_{2}\right)}\right)^{2}+\left(2 \Im x^{\left(r_{1}+r_{2}\right)}\right)^{2}\right\}^{1 / 2} .
\end{aligned}
$$


Thus

$$
d\left({\widetilde{S_{\beta}}}^{k}(\rho(x)),{\widetilde{S_{\beta}}}^{k}(x, \mathbf{0})\right) \leq u^{k} \cdot d(\rho(x),(x, \mathbf{0})) .
$$

From the fact $(x, \mathbf{0}) \in \widehat{Y}$ and $\widetilde{S_{\beta}} \mid \widehat{Y}=\widehat{S_{\beta}}$, we know that

$$
{\widetilde{S_{\beta}}}^{k}(x, \mathbf{0}) \in \widehat{Y} \text {. }
$$

It follows that $\widetilde{S}_{\beta}^{k}(\rho(x))$ comes exponentially close to $\widehat{Y}$ as $k \rightarrow \infty$. By the same reason that we used in the proof of Proposition 5.3, we can conclude that there exists a finite number of $\rho\left(T_{\beta}^{k}\right)$ in a certain bounded domain. Hence

$$
{\widetilde{S_{\beta}}}^{N_{1}}(\rho(x))=\rho\left(T_{\beta}^{N_{1}} x\right) \in \widehat{Y}
$$

for a sufficiently large $N_{1}$. Then $T_{\beta}^{N_{1}} x$ is reduced. From Lemma 5.2 (1), we see that $T_{\beta}^{N} x$ are reduced for any $N \geq N_{1}$.

At last we attain our goal.

TheOREM 5.5. Let $x \in[0,1)$. Then

(1) $x \in \mathbb{Q}(\beta)$ if and only if $x$ has an eventually periodic $\beta$-expansion,

(2) $x \in \mathbb{Q}(\beta)$ is reduced if and only if $x$ has a purely periodic $\beta$-expansion.

Proof. (1) Assume that $x \in \mathbb{Q}(\beta)$. By Proposition 5.4, there exists $N>$ 0 such that $T_{\beta}^{N} x$ is reduced. Proposition 5.3 says that $T_{\beta}^{N} x$ has a purely periodic $\beta$-expansion. Hence $x$ has an eventually periodic $\beta$-expansion. The opposite implication is trivial.

(2) Necessity is obtained by Proposition 5.3. Conversely, assume that $x$ has a purely periodic $\beta$-expansion. According to Proposition 5.4, there exists $N>0$ such that $T_{\beta}^{N} x$ is reduced. The pure periodicity of $x$ implies that there exists $j>0$ such that $T_{\beta}^{N+j} x=x$. Lemma $5.2(1)$ says that $x$ is reduced.

\section{REFERENCES}

[1] S. Akiyama, Pisot numbers and greedy algorithm, Number Theory, Diophantine, Computational and Algebraic Aspects, (K. Györy, A. Pethö and V. T. Sós, eds.), de Gruyter, 1998, pp. 9-21. 
[2] P. Arnoux and Sh. Ito, Pisot substitutions and Rauzy fractals, 8 (2001), Bull. Belg. Math. Soc., 181-207.

[3] A. Bertrand, Développements en base de Pisot et répartition modulo 1, 285 (1977), C.R. Acad. Sci, Paris, 419-421.

[4] A. Brauer, On algebraic equations with all but one root in the interior of the unit circle, Math. Nachr., 4 (1951), 250-257.

[5] F. M. Dekking, Recurrent sets, Adv. in Math., 44 (1982), 78-104.

[6] H. Ei and Sh. Ito, Tilings from characteristic polynomials of $\beta$-expansions, preprint.

[7] C. Frougny and B. Solomyak, Finite beta-expansions, Ergod. Th. and Dynam. Sys., 12 (1992), 713-723.

[8] Y. Hara and Sh. Ito, On real quadratic fields and periodic expansions, Tokyo J. Math., 12 (1989), 357-370.

[9] Sh. Ito, On periodic expansions of cubic numbers and Rauzy fractals, preprint.

[10] Sh. Ito and M. Ohtsuki, Modified Jacobi-Pirron algorithm and generating Markov partitions for special hyperbolic toral automorphisms, Tokyo J. Math., 16 (1993), 441-472.

[11] Sh. Ito and Y. Sano, On periodic $\beta$-expansions of Pisot numbers and Rauzy fractals, Osaka J. Math., 38 (2001), 349-368.

[12] D. Lind and B. Marcus, An Introduction to Symbolic Dynamics and Coding, Cambridge Univ. Press, Cambridge, 1995.

[13] W. Parry, On the $\beta$-expansions of real numbers, Acta Math. Acad. Sci. Hunger., 11 (1960), 401-416.

[14] B. Praggastis, Numeration systems and Markov partitions from self similar tilings, Transactions of the American Mathematical Society, 351 (1999), 3315-3349.

[15] G. Rauzy, Nombres algébriques et substitutions, Bull. Soc. Math. France, 110 (1982), $147-178$.

[16] A. Rényi, Representations for real numbers and their ergodic properties, Acta Math. Acad. Sci. Hunger., 8 (1957), 477-493.

[17] Y. Sano, P. Arnoux, and Sh. Ito, Higher dimensional extensions of substitutions and their dual maps, J. d'Analyse Math., 83 (2001), 183-206.

[18] K. Schmidt, On periodic expansions of Pisot numbers and Salem numbers, Bull. London math. Soc., 12 (1980), 269-278.

[19] B. Solmyak, Dynamics of self-similar tilings, Ergod. Th. \& Dynam. Sys., 17 (1997), 1-44.

Department of Mathematics and Computer Science

Tsuda College

2-1-1 Tsuda-Machi

Kodaira

Tokyo, 187-857\%

Japan

sano@tsuda.ac.jp 